\title{
ANÁLISE DO PERFIL ACADÊMICO DOS COORDENADORES DAS FACULDADES DE MEDICINA NO BRASIL
}

\author{
ANÁLISIS DEL PERFIL ACADÉMICO DE LOS COORDINADORES DE LAS \\ ESCUELAS DE MEDICINA EN BRASIL
}

\section{ANALYSIS OF THE ACADEMIC AND SCIENTIFIC CHARACTERISTICS OF THE BRAZILIANS MEDICAL SCHOOLS COORDINATOR}

\author{
Leonardo Maso NASSAR ${ }^{1}$ \\ Jorge Luís Sanchez AREVALO ${ }^{2}$
}

\begin{abstract}
RESUMO: Este estudo teve por objetivo analisar o perfil acadêmico dos coordenadores do curso de medicina no Brasil. Com base no estudo, visa-se traçar uma ideia da concentração em relação ao tipo de área de pesquisa. A abordagem metodológica usada foi qualitativa e documentaria. Os resultados indicam que existe uma grande concentração em publicações na área de pediatria e ginecologia, quer seja, tanto por universidades públicas e privadas. Ao fazer um comparativo dos últimos 5 anos de pesquisa - medido por publicações de artigos -, observase que a contribuição dos coordenadores das universidades públicas em relação ao total representa o $62 \%$. No geral, observa-se um grande avanço no que concerne ao campo de pesquisa, quer seja, universidade federal, estadual ou privada.
\end{abstract}

PALAVRAS CHAVE: Brasil; Faculdades de medicina; Publicações.

RESUMEN: Este estudio pretendía analizar el perfil académico de los coordinadores del curso de medicina en Brasil. Basándonos en el estudio, pretendemos trazar una idea de la concentración en relación con el tipo de área de investigación. El enfoque metodológico utilizado fue cualitativo y documentado. Los resultados indican que hay una gran concentración en las publicaciones en el área de Pediatría y Ginecología, ya sea por las universidades públicas y privadas. Al comparar los últimos 5 años de investigación-según lo medido por las publicaciones del artículo-se observa que la contribución de los coordinadores de las universidades públicas en relación con el total representa el 62\%. En general, hay un gran progreso en el campo de la investigación, ya sea federal, estatal o privada de la Universidad.

PALABRAS CLAVE: Brasil. Facultades médicas. Publicaciones.

\footnotetext{
${ }^{1}$ Universidade de São Paulo (USP), Ribeirão Preto - SP - Brasil. Doutorando em enfermagem pela Escola de Enfermagem de Ribeirão Preto. ORCID: <https://orcid.org/0000-0002-4300-4293>. E-mail: leo.mnassar@gmail.com.

${ }^{2}$ Universidade de São Paulo (USP), Ribeirão Preto - SP - Brasil. Professor doutor na Universidade Federal de Mato Grosso do Sul (UFMS), Área Finanças / Administração. Pós-doutorado em Administração de Organizações na Universidade de São Paulo (USP) estudando as relações de comércio entre o Brasil e países de América do Sul. ORCID: <https://orcid.org/0000-0002-8426-2096>. E-mail: jsarevalo@gmail.com.
} 
ABSTRACT: This study aimed to analyze the academic profile of the coordinators of the medical course in Brazil. Based on the study, it is intended to draw an idea of the concentration in relation to the type of research area. The methodological approach used was qualitative and documentary. The results indicate that there is a great concentration in publications in the area of pediatrics and gynecology, both by public and private universities. When comparing the last 5 years of research - as measured by article publications -, it is observed that the contribution of the coordinators of public universities in relation to the total represents $62 \%$. In general, there is a great advance in the field of research, whether it is a federal, state or private university.

KEYWORDS: Brazil, medical schools, publications.

\section{Introdução}

Nos últimos anos a oferta para o ensino de medicina no Brasil teve um crescimento significativo, atrelado a esse fator tem-se a instauração do programa "Mais Médicos" em 2013 (BRASIL, 2013). Entre os anos de 2014 a 2017 no Brasil se inauguraram 85 novas faculdades de medicina. Entretanto, apesar do aumento no número disponível de faculdades, a qualidade do ensino ganhou discussão entorno a questões como, as condições de estruturas e equipamentos defasados em hospitais, além da má preparação estudantil para a prática profissional (MOTA, 2014). Atrelado a essas condições, tem-se as mudanças que envolvem métodos alternativos de ensino que precisaram ser adotados devido às alterações éticas no uso de animais e às dificuldades na obtenção de cadáveres para o ensino (ANDRADE, et al. 2015; RUEFF-BARROSO, et al. 2017; BRANDÃO, CARVALHO-FILHO; FERNANDES, 2018).

Todo esse processo observado, tem como finalidade acrescentar melhorias no ensino, ente tal, segundo a Capes - Coordenação de Aperfeiçoamento de Pessoal de Nível Superior, o Brasil ocupa a $13^{\mathrm{a}}$ posição no ranking de produção científica e o impacto das citações aumentou nos últimos anos (CAPES, 2018). A área médica, segundo o relatório, apresentou alta produtividade com excelência em algumas especialidades (CAPES, 2018).

As informações no relatório da CAPES são valiosas porque a assistência médica futura depende da pesquisa científica atual ${ }^{7}$. Por essa razão, há uma tendência cada vez maior para integrar a formação em pesquisa científica na educação médica de graduação, ocorrendo a integração do ensino e da pesquisa científica nos países ocidentais (ABU-ZAID; ALKATTAN, 2013). A pesquisa científica permite que os alunos desenvolvam habilidades básicas de laboratório, competências de pesquisa de alto nível, como pensamento crítico, resolução de problemas, processamento de pensamentos, avaliação sensata, aprendizagem ao longo da vida, 
formulação de hipóteses e metodologia, resultando em interpretação e comunicação de dados, tanto por via oral e textualmente (HOULDEN, 2004).

As faculdades de medicina podem oferecer aos alunos oportunidades de se engajarem em estudos científicos, tendo importância na promoção dos interesses e no desenvolvimento das habilidades de pesquisa dos futuros médicos (SALGUEIRA, et al. 2012). Para tanto, é destacável que a motivação e o compromisso do coordenador do curso afetam a qualidade da organização do curso e o sucesso do aluno (AL-ALWAN, et al. 2015). Os coordenadores funcionam como gerentes e têm papéis mais importantes no desenvolvimento e na implementação de iniciativas, políticas e resultados do curso (STUCKELMAN; ZAVATCHEN; JONES; 2017).

Como líderes em suas instituições, os coordenadores têm poder para influenciar docentes e discentes para alcançar objetivos, como um maior engajamento nas pesquisas científicas (CUMMINGS, et al. 2018). A importância dos coordenadores aumenta em um cenário de constante privatização do ensino médico brasileiro, onde $62 \%$ das faculdades de medicina abertas até 2017 são instituições particulares, a qualidade da formação dos futuros profissionais é questionada (SCHEFFER; DAL POZ; 2015). Com as dificuldades encontradas pelas faculdades para utilizarem cadáveres e animais no ensino, a qualidade da formação do médico estará ainda mais ameaçada sem a devida importância à pesquisa científica.

Dado o contexto apresentado anteriormente o presente estudo pretendeu analisar o perfil acadêmico dos coordenadores das faculdades de medicina no Brasil. Considera-se para tal finalidade analisar a produção acadêmica dos mesmos e agrupara-los seguindo a formação de origem. Ou seja, traçar um perfil descritivo com base na formação acadêmica e publicações de artigos científicos até o ano de 2017. A finalidade é poder traçar uma visão geral da realidade acadêmica, relacionado ao setor saúde, comparando tanto as instituições privadas e públicas. Consequentemente, por meio desse estudo, servir de apoio na discussão em trono a esse tema.

\section{Metodologia e fonte da informação}

O estudo parte de uma abordagem descritiva, para o qual foram analisadas 303 faculdades de medicina cadastradas no Ministério da Educação até o final de 2017 e as publicações científicas dos respectivos coordenadores. As informações sobre os coordenadores foram obtidas com acesso ao site e-Mec do Ministério da Educação do Brasil e as informações com as publicações científicas foram extraídas da Plataforma Lattes, entre os dias 12 e 23 de fevereiro de 2018. 
Se consideraram apenas artigos científicos publicados em revistas (como publicações cientificas) e em periódicos que estavam presentes na seção “Artigos completos publicados em periódicos" divulgados pelos coordenadores em seus respectivos currículos Lattes. Também foram considerados artigos no prelo. As informações foram armazenadas em uma tabela a ser preenchida com o nome da instituição, o tipo de instituição (particular, federal ou estadual), o Estado, a cidade, o nome do coordenador, a especialidade médica, a escola de formação de doutorado, a escola de formação do mestrado, a escola de graduação, a quantidade total de publicações ao longo da carreira e a quantidade de publicações nos últimos cinco anos.

Caso alguma informação estivesse faltando, o espaço da tabela era deixado em branco. Em casos de o coordenador possuir outra formação diferente da medicina, o "campo especialidade médica" era preenchido com os dizeres "não é médico". Para coordenadores com dupla graduação, foram considerados apenas informações referentes a formação médica. Para as titulações de mestrado e doutorado, foram considerados todas formações além da área médica.

Foram considerados como artigos publicados nos últimos cinco anos todas as publicações realizadas de 2012 até a data final da coleta de dados. A opção por selecionar os últimos cinco anos foi feita para evitar distorções provenientes do tempo de carreira de coordenadores mais antigos. Ao todo, quatro instituições foram excluídas por não possuírem coordenadores cadastrados na base do e-Mec nas datas de realização da coleta de dados.

Com a informação coletada, os coordenadores foram classificados com base nas publicações dos últimos cinco anos, em um ranking geral englobando todas as faculdades e em rankings de acordo com o tipo de instituição (federal, estadual e particular). Em caso de empate no número de publicações dos últimos cinco anos, o critério de desempate foi o total de publicações ao longo da carreira.

\section{Descrição e análise dos dados}

O levantamento dos dados referentes dos coordenadores das faculdades de medicina possibilitou a descoberta de informações sobre o perfil acadêmico de tais profissionais. Com os dados levantados, se observou que 118 coordenadores das 299 faculdades de medicina que possuíam coordenadores cadastrados no portal e-Mec não possuíam a titulação máxima de doutorado. Dos 118 coordenadores sem doutorado, apenas 7 eram somente graduados, 36 possuíam a graduação e uma especialização e 75 possuíam pós-graduação no nível de mestrado. 
Com relação ao tipo de instituição coordenadas pelos 118 coordenadores sem doutorado, 74 são de faculdades privadas, 31 de faculdades federais e 13 de faculdades estaduais. Em termos proporcionais relacionados com o total dos cursos de medicina, há um equilíbrio em torno dos $40 \%$ dos cursos sendo coordenadores por profissionais sem a titulação de doutor, sendo a proporção de $37 \%$ para faculdades estaduais, 39\% para faculdades federais e $40 \%$ para faculdades particulares.

Dentre os coordenadores com doutorado, vale destacar que 36 obtiveram tal titulação na Universidade Federal de São Paulo. A segunda instituição que mais formou coordenadores no nível de doutor foi a Universidade de São Paulo com 34. As informações sobre dez principais instituições formadoras de coordenadores com nível de doutorado estão presentes no Gráfico 1.

Gráfico 1 - Instituições formadoras de coordenadores

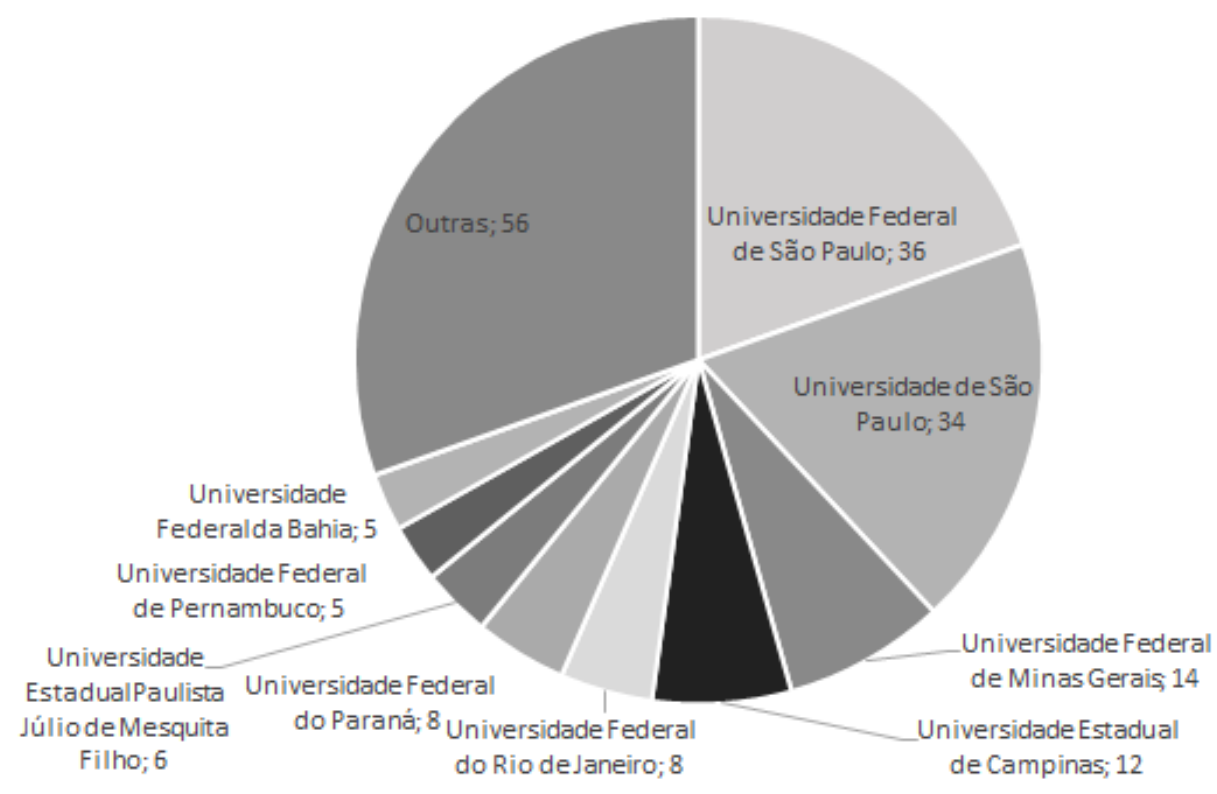

Fonte: Dados coletados (2018) - Autores

Com relação à especialidade médica dos coordenadores, observou-se que 50 eram pediatras, representando aproximadamente $18 \%$ do total de médicos, excluindo da proporção os 16 coordenadores provenientes de outra formação profissional. Além da pediatria, vale destacar a presença de 29 coordenadores ginecologistas, 28 coordenadores especializados em cirurgia geral e 28 especializados em clínica médica. As informações sobre dez principais especialidades dos coordenadores dos cursos de medicina estão presentes no Gráfico 2. 
Gráfico 2 - Especialidades dos coordenadores de medicina

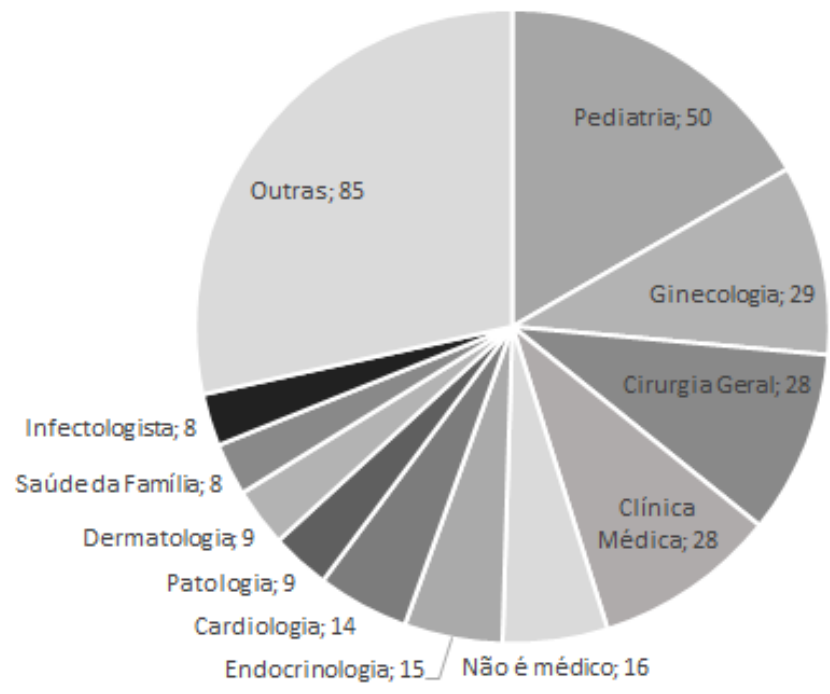

Fonte: Dados coletados (2018) - Autores

Com relação a classificação dos coordenadores baseado nas publicações do último cinco anos, o coordenador que mais teve publicações coordena o curso de medicina da Faculdade de Medicina da Universidade de São Paulo. Nos últimos cinco anos, o coordenador apresentou 172 publicações em periódicos para um total de 727 trabalhos publicados ao longo da carreira. O segundo colocado foi o coordenador do curso de medicina da Faculdades Integradas Pitágoras com 86 trabalhos publicados em periódicos nos últimos cinco anos, sendo um pesquisador bastante ativo recentemente, pois ao longo de toda sua carreira, o coordenador publicou 127 artigos.

Dos dez coordenadores mais bem classificados, oito são coordenam cursos em instituições privadas de ensino. Além do coordenador da Universidade de São Paulo, o único representante de uma instituição pública é o coordenador do curso de medicina da Universidade Federal do Triângulo Mineiro que não é médico e está na quarta colocação. As informações sobre as faculdades dos coordenadores mais bem classificados, a especialidade médica, a escola do doutorado, a escola de graduação e as informações referentes às publicações estão na Figura 1 (Anexo1).

Pela análise dos dados referentes aos coordenadores das faculdades federais de medicina, observa-se que dentre os dez profissionais mais bem classificados no ranking de publicação, $40 \%$ não são médicos. Também é possível auferir que quatro coordenadores devem estar em início de carreira devido a concentração das publicações nos últimos cinco anos, conforme observado pelo coordenador da Universidade Federal de Lavras por exemplo. As 
informações referentes aos coordenadores das faculdades federais de medicina estão presentes na Figura 2 (Anexo 2).

Com relação aos dados referentes aos coordenadores dos cursos de medicina das instituições estaduais, revela-se um predomínio dos profissionais da Universidade de São Paulo, sendo docentes da instituição os três primeiros colocados. Do mesmo modo que ocorreu com alguns coordenadores das faculdades federais, é possível auferir que seis entre os dez coordenadores mais bem colocados devem ter iniciado recentemente a carreira acadêmica devido à concentração das publicações nos últimos cinco anos. As informações referentes aos coordenadores das faculdades estaduais de medicina estão presentes na Figura 3 (Anexo 3).

Já com relação aos dados dos coordenadores de instituições privadas, se ressalta que apenas três docentes entre os dez mais bem colocados possuem menos do que cem publicações ao longo de toda a carreira. Entretanto, dos três coordenadores com menos de cem publicações, dois deles apresentam concentração das publicações nos últimos cinco anos. As informações referentes aos coordenadores das faculdades privadas de medicina estão presentes na Figura 4 (Anexo 4).

Os dados levantados também possibilitaram informações referentes ao tempo das publicações dos coordenadores. Ao todo, $46 \%$ de todos os coordenadores possuem a maioria de suas publicações anteriores a cinco anos, $29 \%$ possuem a maioria de suas publicações com cinco anos ou menos e $26 \%$ não possuem nenhuma publicação científica na carreira. Com relação aos últimos cinco anos, 118 coordenadores, aproximadamente 39\% do total, não realizaram nenhuma publicação científica.

O quadro 1 possui os dados das publicações dos coordenadores com os totais de coordenadores com zero publicações e os totais de coordenadores com mais publicações antigas e com mais publicações novas. Se um coordenador possuía 50\% das suas publicações realizadas nos últimos cinco anos, ele era incluído no grupo com mais publicações novas. Também estão discriminados os totais por nível de titulação para cada um dos tipos de instituição de ensino superior. 
Quadro 1 - Dados de publicação dos coordenadores de medicina

\begin{tabular}{|c|c|c|c|c|}
\hline & $\begin{array}{c}\text { Coordenadores } \\
\text { com Zero } \\
\text { Publicações }\end{array}$ & $\begin{array}{c}\text { Coordenadores } \\
\text { com mais } \\
\text { publicações } \\
\text { antigas }\end{array}$ & $\begin{array}{l}\text { Coordenadores } \\
\text { com mais } \\
\text { publicações novas }\end{array}$ & $\begin{array}{c}\text { Total de } \\
\text { coordenadores }\end{array}$ \\
\hline Estadual & 9 & 16 & 10 & 35 \\
\hline Doutorado & 1 & 13 & 8 & 22 \\
\hline Especialização & 2 & & 1 & 3 \\
\hline Graduação & 1 & & & 1 \\
\hline Mestrado & 5 & 3 & 1 & 9 \\
\hline Federal & 13 & 40 & 26 & 79 \\
\hline Doutorado & 1 & 30 & 17 & 48 \\
\hline Especialização & 8 & 3 & 3 & 14 \\
\hline Graduação & 1 & & 2 & 3 \\
\hline Mestrado & 3 & 7 & 4 & 14 \\
\hline Particular & 31 & 114 & 40 & 185 \\
\hline Doutorado & 6 & 85 & 20 & 111 \\
\hline Especialização & 12 & 3 & 4 & 19 \\
\hline Graduação & 2 & 1 & & 3 \\
\hline Mestrado & 11 & 25 & 16 & 52 \\
\hline TOTAL & 53 & 170 & 76 & 299 \\
\hline
\end{tabular}

Fonte: os autores a partir de dados coletados (2018).

\section{Discussão}

O presente estudo buscou descrever e analisar apenas numericamente as publicações, sem análises sobre a qualidade dos locais das publicações do perfil acadêmico dos coordenadores das faculdades de medicina no Brasil. Para tanto, foram levantados dados acadêmicos de 299 docentes que estavam ocupando o cargo de coordenador entre os dias 12 e 23 de fevereiro de 2018.

Pela análise da classificação que engloba todas as faculdades de medicina, foi percebido um predomínio de coordenadores de instituições privadas de ensino. Um dos fatores que pode explicar a diferença entre as publicações dos coordenadores das instituições privadas e das públicas é o tempo de carreira. Dos dez melhores colocados entre os coordenadores das 
faculdades federais, oito possuem mais da metade de suas publicações realizadas nos últimos cinco anos, sendo seis para faculdades estaduais e apenas quatro para as faculdades particulares.

O tempo de carreira é importante a ser considerado porque o pesquisador consegue desenvolver uma estrutura de pesquisa maior, obtendo maiores chances de publicação. Um dado que pode reforçar tal argumento é o ano de fundação da Faculdade Dinâmica do Vale do Piranga (2015) e da Faculdade Metropolitana São Carlos (2017). Tais faculdades são muito recentes, indicando que os coordenadores possivelmente já trabalharam para outras instituições anteriormente.

Entretanto, vale destacar as informações a respeito dos períodos de publicações entre os coordenadores. Enquanto $33 \%$ dos coordenadores federais e $29 \%$ dos coordenadores estaduais possuem a maior parte das suas publicações científicas realizadas nos últimos cinco anos, a porcentagem cai para $22 \%$ entre os coordenadores particulares. Ao discriminar os dados pelos coordenadores que possuem doutorado, os números aumentam para $36 \%$ entre os coordenadores das estaduais e para $35 \%$ entre os coordenadores das federais com a maior parte das suas publicações científicas realizadas nos últimos cinco anos, entretanto, a porcentagem reduz para $18 \%$ entre os coordenadores das universidades particulares.

Obviamente, com os resultados apresentados é impossível refutar os comentários de Rosenberg (1999), McCowan (2004) e Chaves e Amaral (2016) sobre as instituições de ensino superior privadas desprivilegiarem a pesquisa replicando apenas o conhecimento já produzido anteriormente porque as publicações dos coordenadores não refletem necessariamente a produção científica de toda a instituição. É possível que docentes com mais tempo de carreira sejam contratados para ocuparem os cargos de coordenadores devido à sua experiência de atuação. Entretanto, os dados sugerem que existe potencial para a pesquisa nas instituições privadas por estarem sendo coordenadas por docentes com experiência na produção de conhecimento científico.

Portanto, apesar das limitações do trabalho provenientes da falta de análise da qualidade das publicações e pelo fato do cargo de coordenador ser rotativo, o trabalho contribui para uma a discussão sobre a qualidade das faculdades de medicina no Brasil. Apesar de ser necessária uma análise mais detalhada da qualidade das publicações e do corpo docente como um todo, conclui-se que há potencial para a geração de conhecimento nas faculdades privadas por conta da presença de coordenadores com experiência na geração de conhecimento científico. Desse modo, a fim de trabalhar com as limitações do presente trabalho, sugere-se a investigação mais detalhada das produções científicas de cada coordenador bem como a relação com a geração de conhecimento das instituições coordenadas por eles. 


\section{REFERÊNCIAS}

ABU-ZAID, A.; ALKATTAN, K. Integration of scientific research training into undergraduate medical education: a reminder call. Medical Education Online, v. 18, n. 1, 2013.

AL-ALWAN, I.; BAIG, L. A.; BADRI, M.; MAGZOUB, M. E.; ALYOUSIF, S. Effect of course coordinator behavior and motivation on students' achievement: results from five curriculum blocks of two undergraduate student cohorts at King Saud bin Abdulaziz University of Health Sciences. Pak J Med Sci, v. 31, n. 2, p. 457-461, 2015.

ANDRADE, G. M.; LOPES, H. D. P.; FELÍCIO, S. J. O.; CARMO, V. M.; MATOS, E. P. Experience report on teaching surgical technique without animal use. Acta Cirurgica Brasileira, v. 30, n. 5, p. 371-375, 2015.

BRANDÃO, C. F. S.; CARVALHO-FILHO, M. A.; CECILIO-FERNANDES, D. Simulation centers and pedagogical planning: Two sides of the same coin. Scientia Medica, v. 28, n. 1, 2018 .

BRASIL. Lei $\mathrm{n}^{\circ} 12.871$, de 22 de Outubro de 2013. Institui o Programa Mais Médicos, altera as Leis $\mathrm{n}^{\circ} 8.745$, de 9 de dezembro de 1993, e n 6.932 , de 7 de julho de 1981, e dá outras providências. Diário Oficial da União - Seção 1 - 23/10/2013, Página 1

CAPES. Research in Brazil: a report for CAPES by Clarivate Analytics. Disponível em: http://www.capes.gov.br/images/stories/download/diversos/17012018-CAPES-InCitesReportFinal.pdf. Acesso em: 04 maio 2019.

CHAVES, V. L. J.; AMARAL, N. C. Política de expansão da educação superior no Brasil - O PROUNI e o FIES como financiadores do setor privado. Educ. Rev, v. 32, n. 4, p. 49-72, 2016.

CUMMINGS, G. G.; TATE, K.; LEE, S.; WONG, C. A.; PAANANEN, T.; MICARONI, S. P. M.; CHATTERJEE, G. E. Leadership styles and outcome patterns for the nursing workforce and work environment: a systematic review. International Journal of Nursing Studies, v. 85, n. 1, p. 19-60, 2018.

HOULDEN, R. L.; RAJA, J. B.; COLLIER, C. P.; CLARK, A. F.; WAUGH, J. M. Medical students' perceptions of an undergraduate research elective. Medical Teacher, v. 26, n. 7, p. 659-661, 2004.

MCCOWAN T. The growth of private higher education in Brazil: implications for equity and quality. Journal of Education Policy, v. 19, n. 4, p. 453-472, 2004.

MOTA, A.; CARVALHO, B.; CANDIDO, L.; LOMATO, R.; MAIA T. Exame do CREMESP como indicador da qualidade do ensino médico. Revista Brasileira de Educação Médica, v. 38, n. 1, p. 150-159, 2014. 
RUEFF-BARROSO, C. R.; GARCIA, K. S.; PERUZINI, K. S.; FERNANDES-SANTOS, C.; PEREIRA-SAMPAIO, M. A. Simple and low cost tridimensional model of the cerebral hemisphere. Jornal Internacional de Morfologia, v. 35, n. 2, p. 529-532, 2017.

ROSENBERG, L. E. Physician-scientists - Endangered and essential. Science, n. 283 (5400), p. 331-332, 1999.

SAlGUEIRA, A.; COSTA, P.; GONÇALVES, M.; MAGAlHÃES, E.; COSTA, M. J. Individual characteristics and students engagement in scientific research: a cross-sectional study. BMC Medical Education, v. 12, n. 1, 2012.

SCHEFFER M. C.; DAL POZ M. R. The privatization of medical education in Brazil: Trends and challenges. Human Resources for Health, v. 13, n. 1, 2015.

STUCKELMAN, J.; ZAVATCHEN, S. E.; JONES, S. A. The Evolving Role of the Program Coordinator: Five Essential Skills for the Coordinator Toolbox. Academic Radiology, v. 24, n. 6, p. 725-729, 2017.

\section{Como citar este artigo:}

NASSAR, Leonardo Maso.; AREVALO, Jorge Luís Sanchez. Análise do perfil acadêmico dos coordenadores das faculdades de medicina no Brasil. Revista Ibero-Americana de Estudos em Educação, Araraquara, v. 14, n. 3, p. 1066-1080, jul./set., 2019. e-ISSN: 1982-5587. DOI: $10.21723 /$ riaee.v14i3.11892

Data de Submissão: $11 / 11 / 2018$

Revisões requeridas: 04/12/2018

Aceite em: 30/01/2019

Publicado em: 23/03/2019 


\section{Anexo 1}

Figura 1 - Classificação dos coordenadores dos cursos de medicina no Brasil

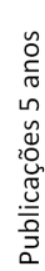

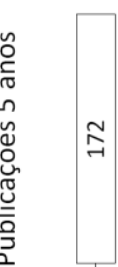
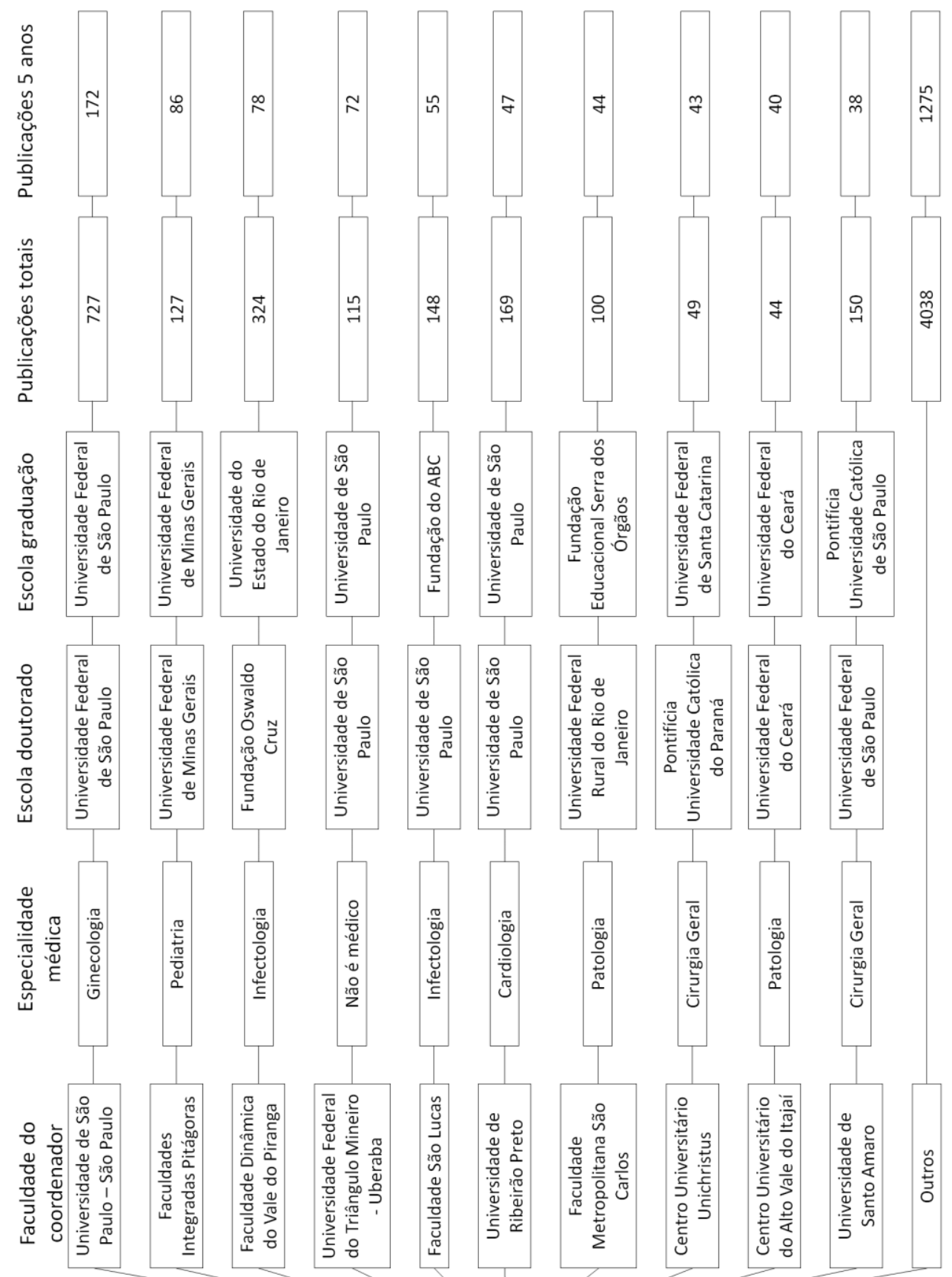


\section{Anexo 2}

Figura 2 - Classificação dos coordenadores das faculdades federais de medicina

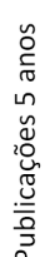
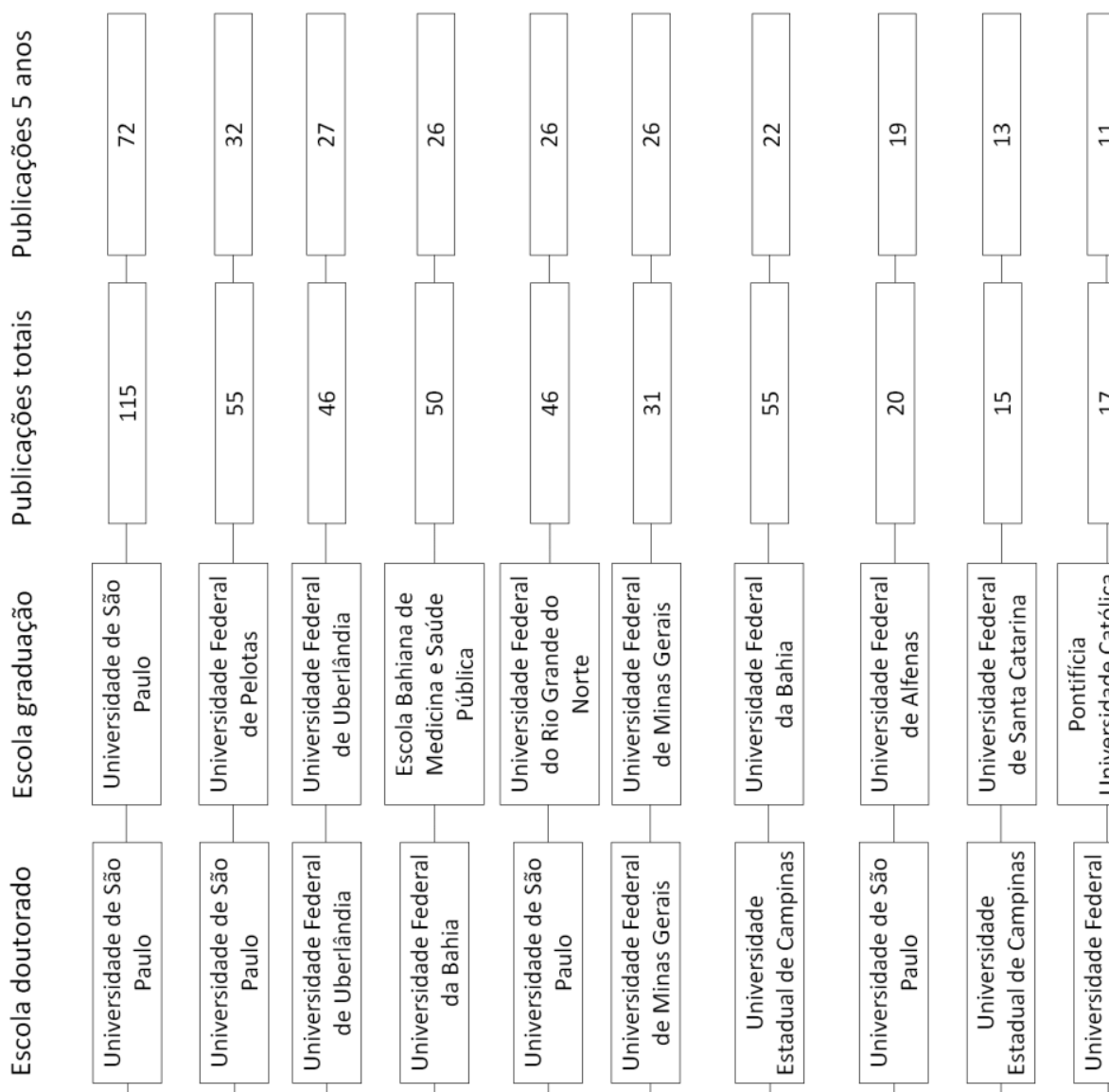

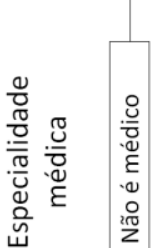
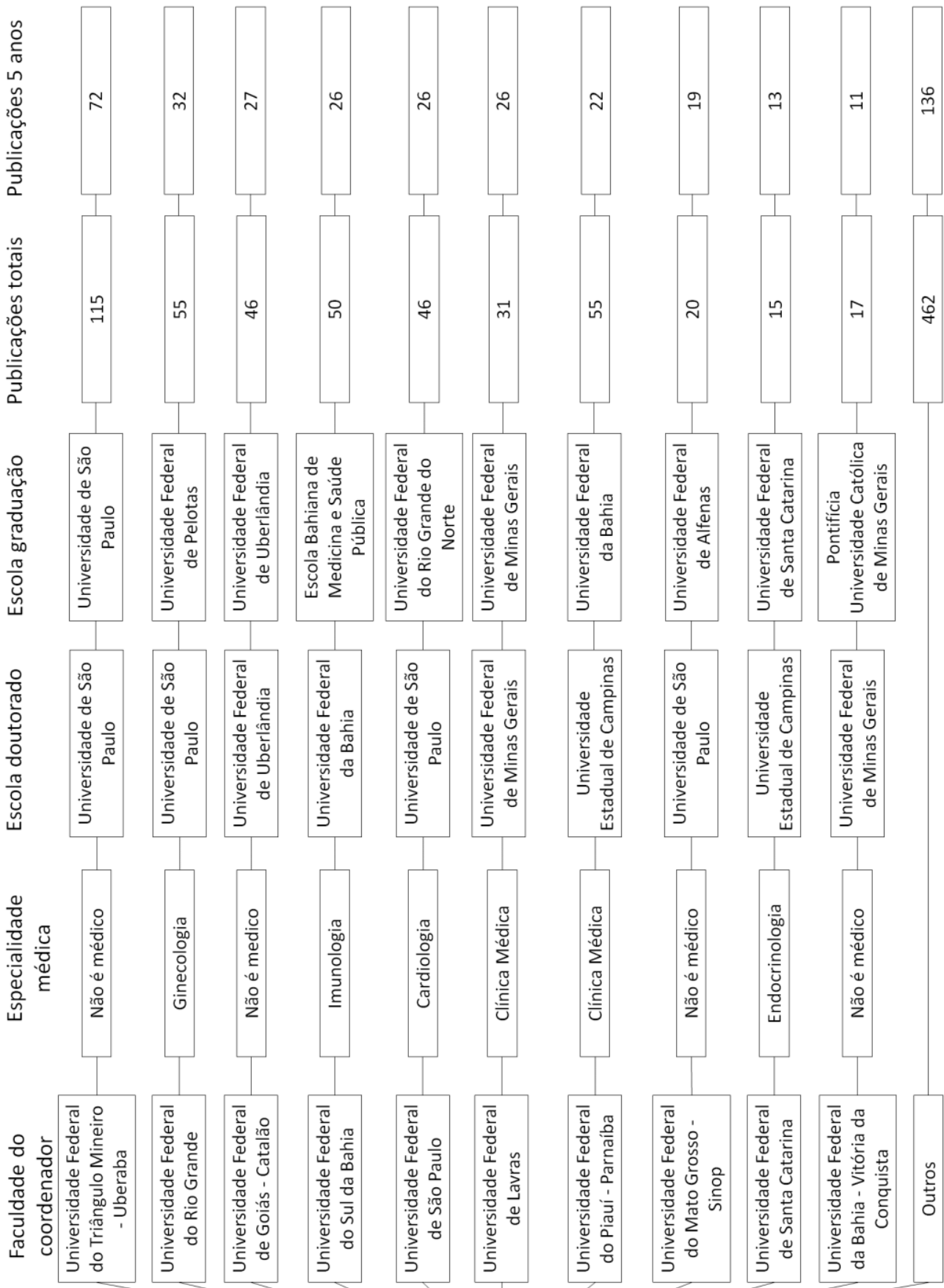

Fonte: Dados coletados (2018) - autores 


\section{Anexo 3}

Figura 3 - Classificação dos coordenadores das faculdades estaduais de medicina

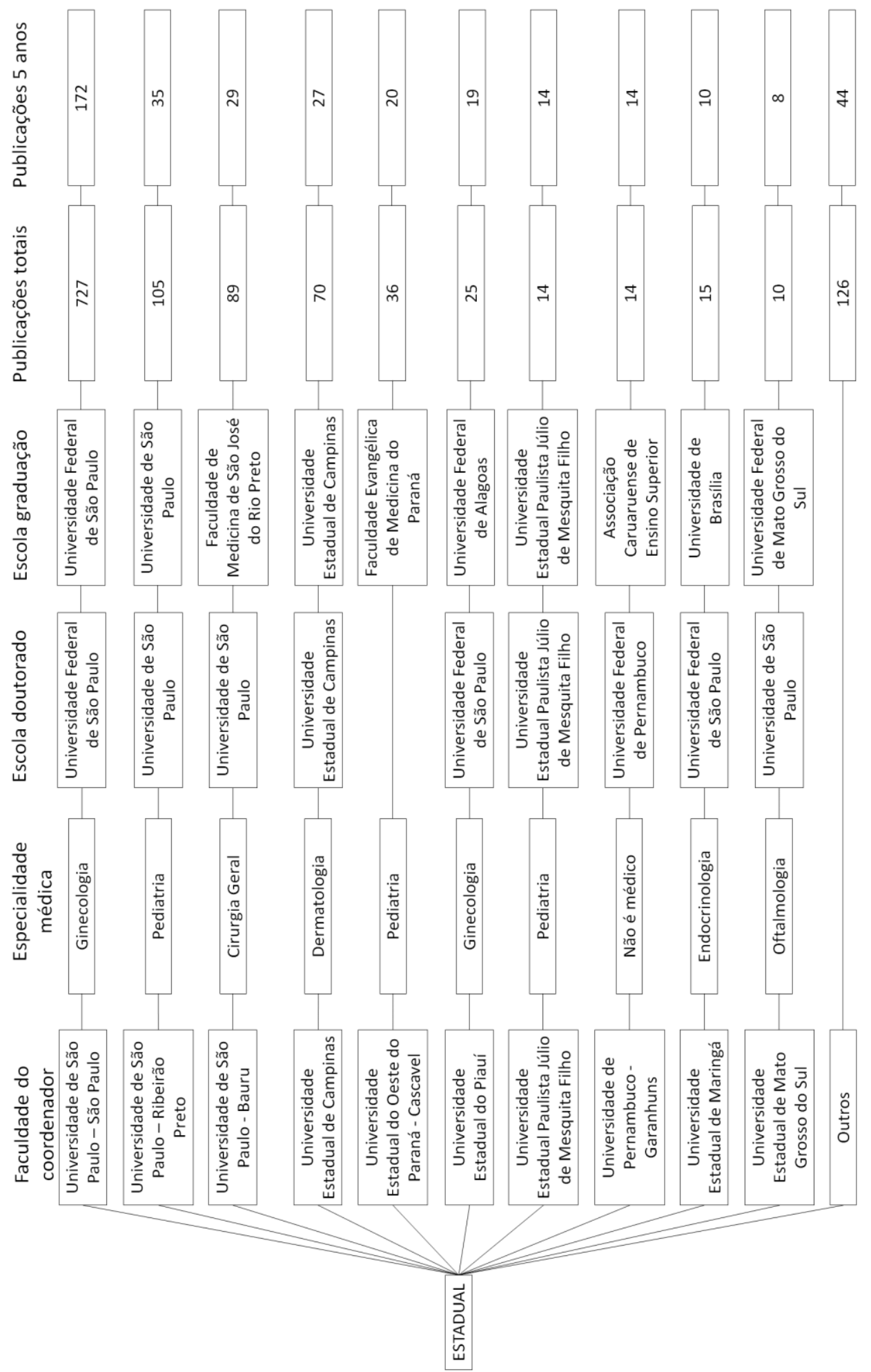

Fonte: Dados coletados (2018) - autores 


\section{Anexo 4}

Figura 4 - Classificação dos coordenadores das faculdades privadas de medicina

ปั

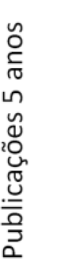
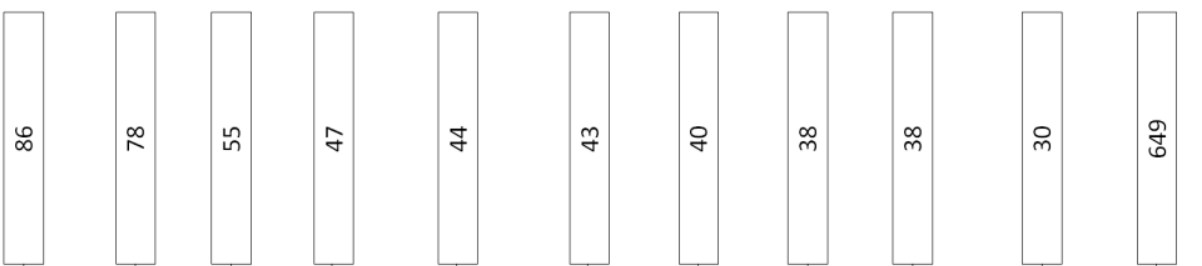

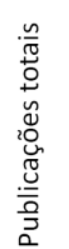
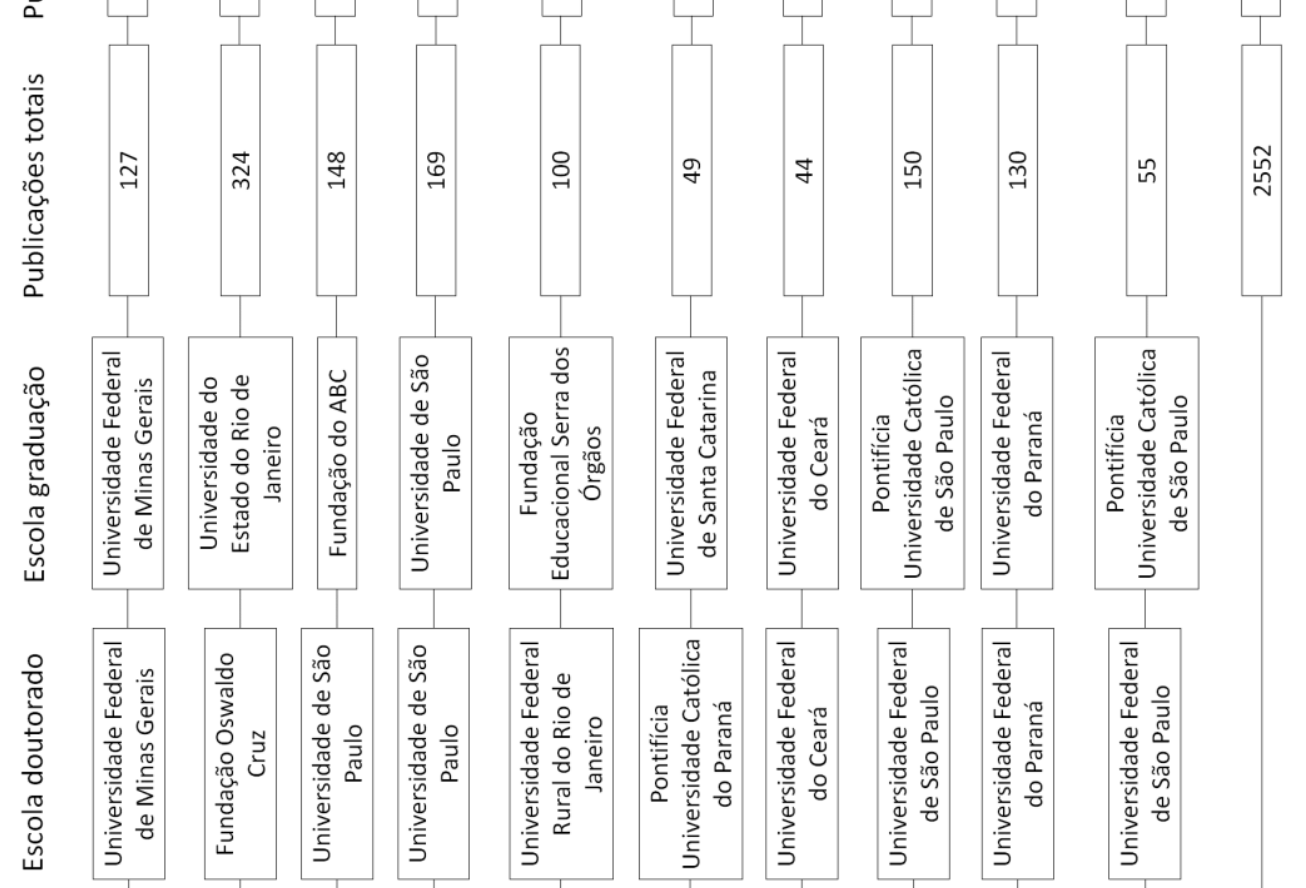

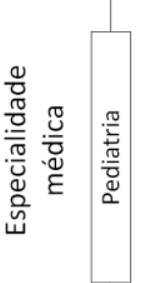
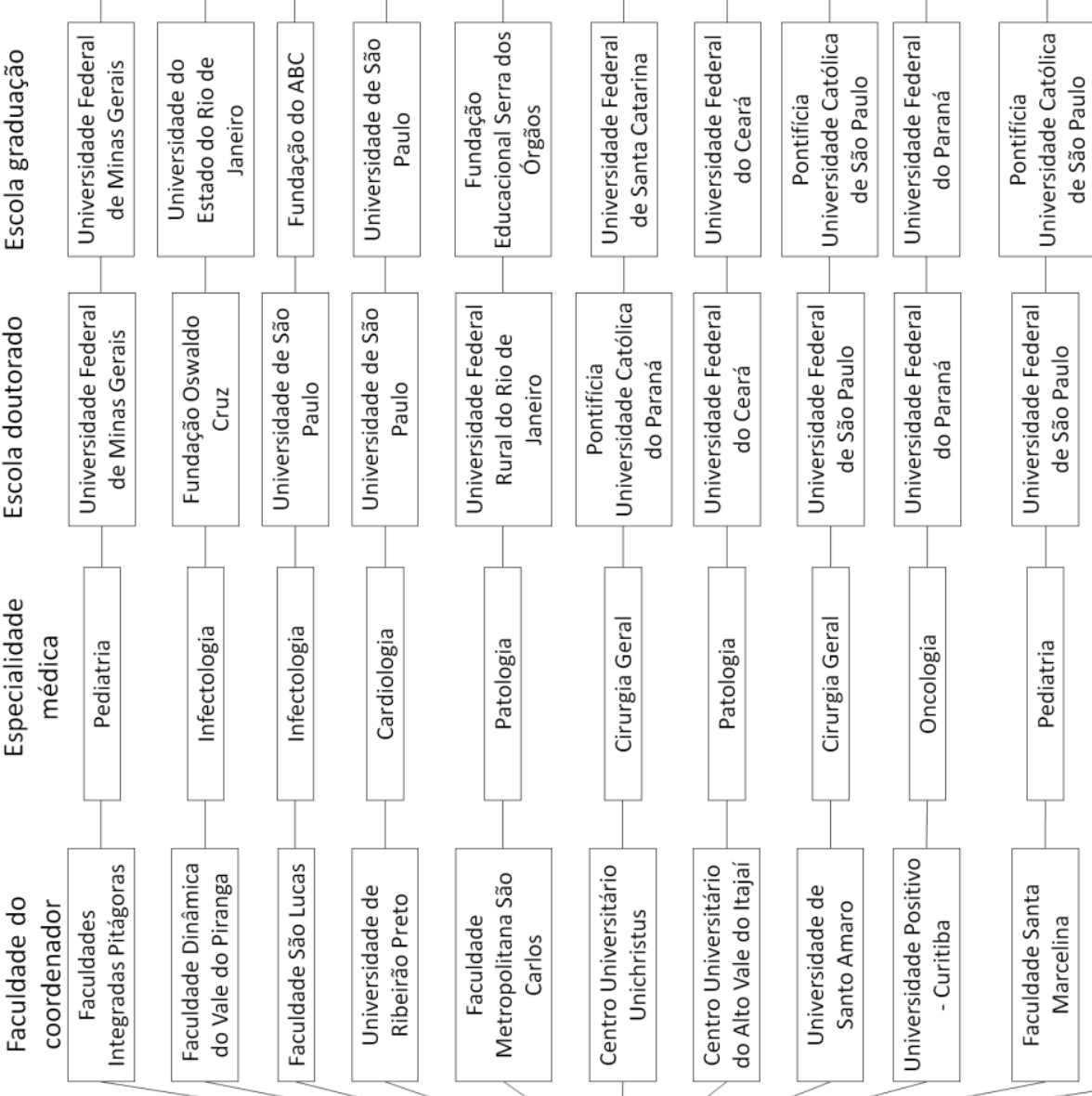

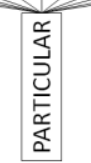

Fonte: Dados coletados (2018) - autores 